\title{
A systematic review of dose-volume predictors and constraints for late bowel toxicity following pelvic radiotherapy
}

\author{
Rashmi Jadon ${ }^{1,2^{*}}$, Emma Higgins', Louise Hanna ${ }^{1}$, Mererid Evans ${ }^{1}$, Bernadette Coles ${ }^{3}$ and John Staffurth ${ }^{1,4}$
}

\begin{abstract}
Background: Advanced pelvic radiotherapy techniques aim to reduce late bowel toxicity which can severely impact the lives of pelvic cancer survivors. Although advanced techniques have been largely adopted worldwide, to achieve their aim, knowledge of which dose-volume parameters of which components of bowel predict late bowel toxicity is crucial to make best use of these techniques.

The rectum is an extensively studied organ at risk (OAR), and dose-volume predictors of late toxicity for the rectum are established. However, for other components of bowel, there is a significant paucity of knowledge. The Quantitative Analyses of Normal Tissue Effects in the Clinic (QUANTEC) reviews recommend dose-volume constraints for acute bowel toxicity for peritoneal cavity and bowel loops, although no constraints are recommended for late toxicity, despite its relevance to our increasing number of survivors. This systematic review aims to examine the published literature to seek dose-volume predictors and constraints of late bowel toxicity for OARs (apart from the rectum) for use in clinical practice.
\end{abstract}

Methods: A systematic literature search was performed using Medline, Embase, Cochrane Library, Web of Science, Cinahl and Pubmed. Studies were screened and included according to specific pre-defined criteria. Included studies were assessed for quality against QUANTEC-defined assessment criteria.

Results: 101 studies were screened to find 30 relevant studies. Eight studies related to whole bowel, 11 to small bowel, and 21 to large bowel (including 16 of the anal canal). The anal canal is an important OAR for the development of late toxicity, and we recommend an anal canal Dmean <40Gy as a constraint to reduce late incontinence. For other components of bowel (sigmoid, large bowel, intestinal cavity, bowel loops), although individual studies found statistically significant parameters and constraints these findings were not corroborated in other studies.

Conclusions: The anal canal is an important OAR for the development of late bowel toxicity symptoms. Further validation of the constraints found for other components of bowel is needed. Studies that were more conclusive included those with patient-reported data, where individual symptom scores were assessed rather than an overall score, and those that followed statistical and endpoint criteria as defined by QUANTEC.

\footnotetext{
* Correspondence: Rashmi.jadon@addenbrookes.nhs.uk

${ }^{1}$ Department of Clinical Oncology, Velindre Cancer Centre, Velindre Road,

Whitchurch, Cardiff CF14 2TL, UK

Department of Clinical Oncology, Addenbrookes' Hospital, Box 193,

Cambridge CB2 OQQ, UK

Full list of author information is available at the end of the article
}

(c) The Author(s). 2019 Open Access This article is distributed under the terms of the Creative Commons Attribution 4.0 International License (http://creativecommons.org/licenses/by/4.0/), which permits unrestricted use, distribution, and reproduction in any medium, provided you give appropriate credit to the original author(s) and the source, provide a link to the Creative Commons license, and indicate if changes were made. The Creative Commons Public Domain Dedication waiver (http://creativecommons.org/publicdomain/zero/1.0/) applies to the data made available in this article, unless otherwise stated. 


\section{Background}

Pelvic radiotherapy is used to treat approximately 17,000 patients per year in the UK with urological, gynaecological and colorectal malignancies [1]. For a significant proportion of these patients, pelvic radiotherapy improves survival outcomes. For others, it reduces the risk of pelvic recurrences, which can both cause distressing symptoms and be difficult to manage.

Although contributing to the cure of many pelvic cancer survivors, pelvic radiotherapy is associated with late toxicity, in particular late bowel toxicity. Serious life-threatening toxicity such as bowel obstruction, fistulae and bleeding requiring transfusion occur in $4-10 \%$ of patients 5-10 years after treatment [2]. Furthermore, an important consideration for the growing number of survivors of pelvic cancers is that $50 \%$ of patients report late bowel toxicity symptoms which adversely affect their quality of life after pelvic radiotherapy.

Late bowel toxicity is generally attributed to radiation to bowel and rectum and these are considered the organs at risk (OARs). Advanced radiotherapy techniques for pelvic treatments are continually evolving, with the aim of reducing dose to these OARs.

However, to determine whether the dose reductions achieved by these techniques are likely to translate into reduced toxicity for patients requires detailed knowledge of the dose-volume parameters and constraints for these OARs. Once dose-volume constraints are known these can be used to limit the risk of toxicity and potentially allow safe dose escalation with modern delivery techniques.

In 2010 the Quantitative Analysis of Normal Tissue Effects in the Clinic (QUANTEC) review summarised the available dose-volume data for bowel toxicity, with one review focussing on rectum and the other on stomach and bowel. For rectum, an extensively studied OAR, QUANTEC reviewed a large amount of high-quality data and dose-volume constraints for rectum for acute and late toxicity were recommended [3]. These are commonly incorporated into radiotherapy protocols in clinical practice.

However, for bowel there was a relative paucity of data. QUANTEC reviewed data from six papers which examined the dose-volume relationship of bowel with acute bowel toxicity only [4]. For late bowel toxicity, there was no detailed dose-volume relationship analysis described. Studies mentioned were trial data detailing the incidence of late bowel toxicity at the dose-fractionations used within each trial, though no specific dose-volume predictors can be derived from this information.

The QUANTEC reviewers suggest that the constraints identified for acute bowel toxicity may be applied for late bowel toxicity however clarify that "this correlation is not established". Further, although QUANTEC examined the peritoneal cavity and small bowel loops as OARs, the potential of other bowel components as OARs for bowel toxicity such as sigmoid, duodenum, ileum and anal canal are not detailed.

In a separate paper by Jackson et al., QUANTEC [5] highlighted issues which hinder the development of dose-volume constraints and the pooling of results from different studies, including variations in toxicity endpoint definition, statistical standards, and anatomical definitions of OARs. They recommended several criteria to assess the quality of future dose-volume studies and to facilitate meta-analysis of these studies.

With reduction of late bowel toxicity being a prime aim of advanced pelvic radiotherapy techniques, the lack of clear dose-volume constraints in this setting has been acknowledged and more studies have been reported. This study aims to systematically review published studies examining the dose-volume predictors of all components of bowel (excluding rectum) for late bowel toxicity, including a quality assessment of these studies from criteria derived from QUANTEC.

From this review we aim to determine the clinically useful dose-volume constraints for late bowel toxicity which can guide protocols for advanced pelvic radiotherapy techniques.

\section{Methods}

\section{Information sources and search strategy}

A systematic search was carried out using Medline, Premedline, Embase, Pubmed and Web of Science on 15th October 2013; Updated searches were performed on 10th November 2014, 3rd September 2015 and 1st May 2017 to ensure all new literature was included. Thesaurus and natural language terms around the concepts of "radiotherapy, radiotherapy injuries, side effects, toxicity, intestines bowel, dose, dose fractionation, dose response relationship" were identified for each database. Duplicate references were removed.

\section{Study selection}

Eligible studies were English language studies, involving human adult patients treated for any gastrointestinal, urological or gynaecological malignancies with external beam radiotherapy. Studies correlating the dose-volume relationship of any component of bowel from duodenum to anal canal with late bowel toxicity were included, apart from those focussed on the rectum, given that it has already been extensively studied as an OAR. Late toxicity was defined as more than 3 months from completion of radiotherapy.

Excluded studies were review articles and letters, studies involving brachytherapy only, or stereotactic body radiotherapy. Both full text papers and conference 
abstracts were considered, however studies with insufficient methodological detail to be able to repeat the method on an independent sample of patients were excluded.

All abstracts were independently screened by two reviewers (RJ, EH) for inclusion. Full papers of abstracts were acquired and further assessed for eligibility, with any discrepancies discussed between the two reviewers. The reference lists of all the included papers were hand-searched for additional references.

\section{Data extraction and synthesis of results}

Bowel can be defined in several different ways and for the purpose of this review studies were divided into those looking at the whole bowel (including bowel loops and peritoneal cavity), small bowel (and its components) and large bowel (and its components). For each included study the number of patients, proportion with the toxicity, tumour site, OAR studied, toxicity definition, treatment details and key findings were tabulated.

Furthermore, the recommendations from QUANTEC [5] on quality of dose-volume studies were reviewed, and those criteria that can be applied to this subject were selected (see Table 1). Each included study was assessed for quality against these statistical and endpoint criteria.

Table 1 Statistical and Endpoint Considerations from QUANTEC [5]

\footnotetext{
Statistical considerations

1 Basic statistical data provided on incidence of toxicity

-Both number of subjects and number of events should be reported

-If an estimate of incidence is given the standard error should be supplied

2 Numerical labeling of response histogram - if into groups eg. quartiles must state number of patients in each quartile

3 When predictive models are correlated with complications parameter estimates must be stated with their standard error

4 Complication rates associated with constraints must be reported

5 "Goodness of fit" to be reported such as Chi-squared

6 Discriminator statistics reported such as receiver operating characteristic curves

7 Full organ volumes (rather than partial) should be used

-If this is not possible absolute volumes should be used or a standard method of normalization

-A clear statement of organ volume definition should be given

Toxicity Endpoint considerations

1 Symptom-specific information rather than a portmanteau endpoint (eg. RTOG gr 2) should be used

2 Consideration that symptoms may be attributed to pre-radiotherapy co-morbidities

3 Patient-reporting of symptoms may be important
}

\section{Results}

Outcomes of the systematic search are shown in Fig. 1. Overall, 30 studies involving a total of 5126 patients were included as detailed in Table 2 . Twenty-one studies included patients with prostate cancer, 6 with gynaecological cancers (cervical and endometrial), and 2 each included bladder and pancreatic cancers. Most studies ( $n$ $=18$ ) included less than 100 patients, with 9 studies having less than 50 patients included.

Table 3 details studies for whole and small bowel, and Table 4 those for large bowel. In each table the final two columns indicate the quality assessment criteria of statistical and endpoint considerations as defined in Table 1. If a specific criterion is met its number is noted in the column.

\section{Whole bowel}

Eight papers (including 445 patients) examined the dose-volume relationship of whole bowel either using bowel loops or intestinal/peritoneal cavity as detailed in Table 3.

\section{Peritoneal cavity}

Late bowel toxicity was associated with low doses to the peritoneal cavity (V10-30Gy) in 2 studies. Mouttett-Audouard et al. [6] found, in 37 cervical cancer patients, an association between "bigger volumes" of bowel receiving 10-30Gy and grade 1-3 Common Terminology Criteria Adverse Events (CTCAE) toxicity, although specific cut-offs were not reported. Deville et al. [7] found that peritoneal cavity volume and V20 were both associated with grade 1 Radiation Therapy Oncology Group (RTOG) toxicity. Again no constraints were derived.

\section{Bowel loops}

Two studies $[8,9]$ investigated bowel loops as an OAR for late toxicity, both with an identical definition of bowel loops. Guerrero-Urbano et al., in 79 patients who had their prostate and pelvic nodes treated, found V40, V45 and V60 bowel loops to be predictive of late grade 2 RTOG-graded diarrhoea. They suggested constraints of $\mathrm{V} 40<124 \mathrm{cc}, \mathrm{V} 45<71 \mathrm{cc}$ and $\mathrm{V} 60<0.5 \mathrm{cc}$ to reduce grade 2 RTOG toxicity, although no complication rates associated with these constraints are detailed. McDonald et al. in their study of 47 bladder cancer patients suggested constraints to reduce the risk of grade $\geq 2$ RTOG toxicity to less than $25 \%(\mathrm{~V} 30<178 \mathrm{cc} ; \mathrm{V} 40<151 \mathrm{cc}$; $\mathrm{V} 45<139 \mathrm{cc} ; \mathrm{V} 60<98 \mathrm{cc}$ and $\mathrm{V} 65<40 \mathrm{cc}$ ), although it must be noted that only 3 patients within this study had grade 2 toxicity.

\section{Small bowel and its components}

Eleven studies (including 1401 patients) were included in this section, with 6 studies examining small bowel 


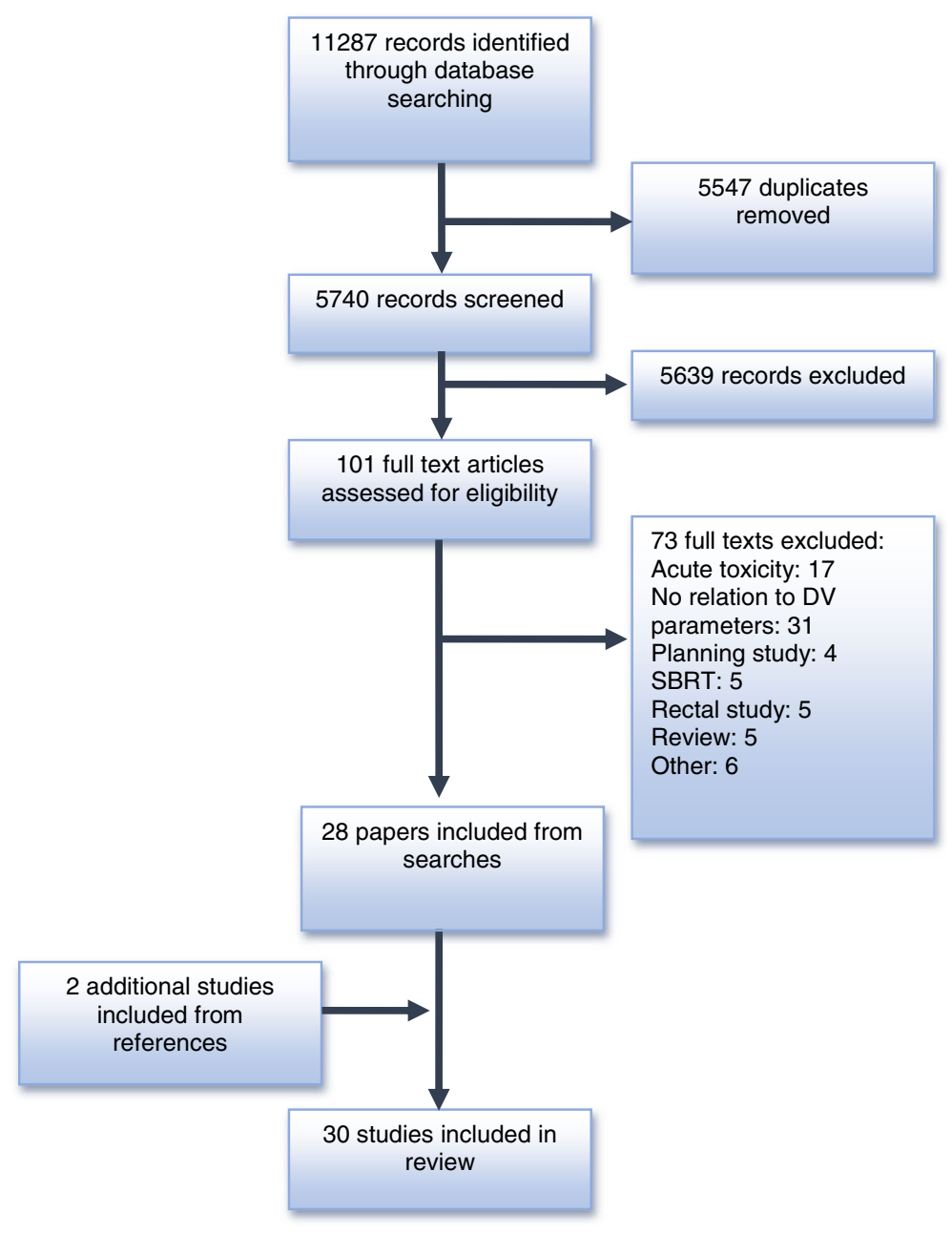

Fig. 1 Systematic Search Outcomes

and 4 examining the duodenum, as detailed in Table 3. No papers investigating the ileum or jejunum were found.

\section{Small bowel}

2 of 6 studies found positive correlations with late bowel toxicities and small bowel volume parameters in cervical cancer patients. However, the positive parameters were different between the studies, with Isohashi et al. [10] recommending a V40 $<340 \mathrm{cc}$, and Chopra et al. [11] recommending a V15 $<275 \mathrm{cc}$. Lind et al. [12] found that a mean small bowel dose $>50$ Gy was of significance, however could not clarify whether toxicity was linked specifically to small bowel, sigmoid or anal sphincter dose, making these results difficult to interpret.

\section{Duodenum}

3 of 4 studies found positive correlations between dose-volume parameters and duodenal toxicity. Two studies found V55 to be an important predictor, though with differing constraints. Kelly et al, in 106 pancreatic patients recommending a V55 $<1 \mathrm{cc}$ [13] and Verma et al. in 105 gynaecological patients recommending a V55 $<15$ cc [14]. Huang et al. [15] found V25 to be the significant predictor for pancreatic cancer patients treated with concurrent gemcitabine; with a V $25<45 \%$ toxicity rates were $8 \%$, above this constraint toxicity was $48 \%$. Investigation of individual duodenal segments did not reveal any positive findings [16].

\section{Large bowel and its components}

21 studies (including 5006 patients) were included in this section (see Table 4), with 2 examining large bowel, 3 examining sigmoid and 16 studies examining the anal canal/sphincter region.

\section{Large bowel and sigmoid Colon}

Chopra et al. [11] found on multivariate analysis that V15 of large bowel was associated with grade 3 CTCAE toxicity $(p<0.03)$, and recommended with the use of the 
Table 2 All included studies

\begin{tabular}{|c|c|c|c|c|c|c|c|c|c|c|}
\hline Author & Year & Cancer site & $\begin{array}{l}\text { No } \\
\text { of } \\
\text { pts }\end{array}$ & Pts with tox & OAR studied & $\begin{array}{l}\text { Toxicity score } \\
\text { used }\end{array}$ & RT Type & $\begin{array}{l}\text { Primary RT } \\
\text { Dose (Gy/\#) }\end{array}$ & $\begin{array}{l}\text { Pelvic RT } \\
\text { dose (Gy/ } \\
\#)\end{array}$ & $\begin{array}{l}\text { Concurrent } \\
\text { chemo use }\end{array}$ \\
\hline $\begin{array}{l}\text { Adkison } \\
{[34]}\end{array}$ & 2012 & Prostate & 53 & 20 & small bowel & CTCAE v3.0 & IMRT & $70 / 28$ & $56 / 28$ & no \\
\hline $\begin{array}{l}\text { al-Abany } \\
{[18]}\end{array}$ & 2005 & Prostate & 65 & 9 & $\begin{array}{l}\text { anal } \\
\text { sphincter } \\
\text { region }\end{array}$ & $\begin{array}{l}\text { Own } \\
\text { questionnaire }\end{array}$ & $3 \mathrm{D}$ & $70.2 / 39$ & NS & no \\
\hline $\begin{array}{l}\text { Alsadius } \\
\text { [19] }\end{array}$ & 2012 & $\begin{array}{l}\text { Prostate or } \\
\text { prostatic bed }\end{array}$ & 403 & 51 & $\begin{array}{l}\text { anal } \\
\text { sphincter } \\
\text { region }\end{array}$ & $\begin{array}{l}\text { Own } \\
\text { questionnaire }\end{array}$ & $3 \mathrm{D}$ & $70 / 35$ & NS & no \\
\hline $\begin{array}{l}\text { Buettner } \\
{[20]}\end{array}$ & 2012 & Prostate & 388 & 57 & anal canal & $\begin{array}{l}\text { Common } \\
\text { grading scheme }\end{array}$ & $3 \mathrm{D}$ & $64 / 32$ or $74 / 37$ & NS & no \\
\hline Chopra [11] & 2014 & $\begin{array}{l}\text { Cervix (post- } \\
\text { op) }\end{array}$ & 71 & 9 & $\begin{array}{l}\text { small bowel, } \\
\text { large bowel }\end{array}$ & CTCAE v3.0 & $\begin{array}{l}\text { IG-MRT (46); } \\
\text { 3D (25) }\end{array}$ & $50 / 25$ & $50 / 25$ & $\begin{array}{l}\text { 63/71 } \\
\text { cisplatin }\end{array}$ \\
\hline Deville [31] & 2010 & Prostate & 30 & 2 & $\begin{array}{l}\text { intestinal } \\
\text { cavity }\end{array}$ & RTOG & IMRT & $79.2 / 44$ & $45 / 25$ & no \\
\hline Deville [7] & 2012 & Prostatic bed & 36 & 5 & $\begin{array}{l}\text { intestinal } \\
\text { cavity }\end{array}$ & RTOG & IMRT & $70.2 / 39$ & $45 / 25$ & no \\
\hline Ebert [35] & 2015 & Prostate & 754 & $\begin{array}{l}\text { Symptom } \\
\text { specific }\end{array}$ & Anal canal & LENT-SOMA & IMRT & $66-78 / 33-38$ & NS & no \\
\hline Fokdal [14] & 2005 & $\begin{array}{l}\text { Prostate or } \\
\text { bladder }\end{array}$ & 71 & $\begin{array}{l}\text { Symptom } \\
\text { specific }\end{array}$ & small bowel & LENT-SOMA & Conformal & $\begin{array}{l}\text { 60/30 (bladder) } \\
69.6 / 35 \\
\text { (prostate) }\end{array}$ & $\begin{array}{l}\text { 48-60Gy } \\
\text { bladder; } \\
\text { NS for } \\
\text { prostate) }\end{array}$ & no \\
\hline $\begin{array}{l}\text { Fonteyne } \\
{[17]}\end{array}$ & 2007 & Prostate & 241 & $\begin{array}{l}\text { Symptom } \\
\text { specific }\end{array}$ & $\begin{array}{l}\text { small bowel, } \\
\text { sigmoid }\end{array}$ & $\begin{array}{l}\text { RTOG and } \\
\text { "RILIT" }\end{array}$ & IMRT & $74 / 37-80 / 40$ & NS & no \\
\hline Green [36] & 2015 & $\begin{array}{l}\text { Prostate or } \\
\text { prostatic bed }\end{array}$ & 73 & 10 & $\begin{array}{l}\text { Intestinal } \\
\text { cavity }\end{array}$ & CTCAE v4.0 & IMRTNMMAT & $61-79.2$ & 45 & no \\
\hline $\begin{array}{l}\text { Guerrero- } \\
\text { Urbano [8] }\end{array}$ & 2010 & $\begin{array}{l}\text { Prostate \& } \\
\text { Pelvic nodes }\end{array}$ & 79 & 21 & bowel loops & $\begin{array}{l}\text { RTOG diarrhoea } \\
\& \text { LENT SOMA } \\
\text { diarrhoea }\end{array}$ & IMRT & $70 / 35$ & $\begin{array}{l}50 / 35 \text { or } \\
55 / 35\end{array}$ & no \\
\hline Huang [15] & 2011 & Pancreas & 46 & 8 & duodenum & CTCAE v4.0 & $3 \mathrm{D}$ or IMRT & $42 / 15$ & $\begin{array}{l}42 / 15 ; 36 / \\
15 ; 38 / 19\end{array}$ & $\begin{array}{l}\text { Gemcitabine; } \\
18 \text { pts. } \\
\text { erlotinib in } \\
\text { addition }\end{array}$ \\
\hline $\begin{array}{l}\text { Isohashi } \\
{[10]}\end{array}$ & 2013 & $\begin{array}{l}\text { Cervix (post- } \\
\text { op) }\end{array}$ & 97 & 16 & $\begin{array}{l}\text { peritoneal } \\
\text { cavity, small } \\
\text { and large } \\
\text { bowel }\end{array}$ & RTOG/EORTC & $2 \mathrm{D}$ or $3 \mathrm{D}$ & $50 / 25$ & $50 / 25$ & $\begin{array}{l}\text { All } \\
\text { nedaplatin }\end{array}$ \\
\hline Kelly [13] & 2013 & Pancreas & 106 & 20 & duodenum & CTCAE V4.0 & 3D or IMRT & $\begin{array}{l}50.4 / 28 \text { (78pts); } \\
57.5-75.4 \text { in } \\
28-39 \# \text { (28pts) }\end{array}$ & $\begin{array}{l}50.4 / 28 \\
\text { (78pts); } \\
57.5-75.4 \\
\text { in 28-39\# } \\
\text { (28pts) }\end{array}$ & $\begin{array}{l}\text { Gemcitabine } \\
5 \text {-FUor } \\
\text { capecitabine } \\
+/- \\
\text { cetuximab or } \\
\text { erlotinib }\end{array}$ \\
\hline Author & Year & Cancer site & $\begin{array}{l}\text { No } \\
\text { of } \\
\text { pts }\end{array}$ & Pts with tox & OAR studied & $\begin{array}{l}\text { Toxicity score } \\
\text { used }\end{array}$ & RT Type & RT Dose (Gy/\#) & $\begin{array}{l}\text { Pelvic RT } \\
\text { dose (Gy/ } \\
\#)\end{array}$ & $\begin{array}{l}\text { Concurrent } \\
\text { chemo use }\end{array}$ \\
\hline Koper [25] & 2004 & Prostate & 266 & 141 & anal canal & $\begin{array}{l}\text { RTOG } \\
\text { (simplified) } \\
\text { Symptom } \\
\text { questionnaire }\end{array}$ & $3 \mathrm{D}$ or $2 \mathrm{D}$ & $66 / 33$ & NS & no \\
\hline Lind [12] & 2016 & $\begin{array}{l}\text { Cervical or } \\
\text { Endometrium }\end{array}$ & 519 & 63 & $\begin{array}{l}\text { Anal } \\
\text { sphincter, } \\
\text { small bowel, } \\
\text { sigmoid }\end{array}$ & $\begin{array}{l}\text { Own } \\
\text { questionnaire } \\
\text { (defecation into } \\
\text { clothing } \\
\text { without } \\
\text { forewarning) }\end{array}$ & $2 \mathrm{D}$ or $3 \mathrm{D}$ & $\begin{array}{l}40-46 \\
\text { (endometrium) } \\
\text { or } 55-70 \\
\text { (cervix) }\end{array}$ & NS & Not stated \\
\hline
\end{tabular}


Table 2 All included studies (Continued)

\begin{tabular}{|c|c|c|c|c|c|c|c|c|c|c|}
\hline $\begin{array}{l}\text { Mavroidis } \\
{[27]}\end{array}$ & 2005 & Prostate & 65 & $\begin{array}{l}\text { Symptom } \\
\text { specific }\end{array}$ & $\begin{array}{l}\text { anal } \\
\text { sphincter }\end{array}$ & $\begin{array}{l}\text { Own } \\
\text { questionnaire }\end{array}$ & $3 \mathrm{D}$ & $70.2 / 39$ & NS & no \\
\hline $\begin{array}{l}\text { Mcdonald } \\
{[9]}\end{array}$ & 2015 & Bladder & 47 & 10 & bowel loops & RTOG & $3 D$ & $64 / 32$ & $64 / 32$ & $\begin{array}{l}21 \text { received } \\
\text { 5-FU/MMC }\end{array}$ \\
\hline $\begin{array}{l}\text { Mouttet- } \\
\text { Audouard } \\
{[6]}\end{array}$ & 2015 & Cervical & 37 & 8 & $\begin{array}{l}\text { Small bowel } \\
\text { [defined as } \\
\text { peritoneal } \\
\text { cavity], } \\
\text { sigmoid }\end{array}$ & CTCAE V4.0 & $\begin{array}{l}\text { IMRT } \\
\text { (tomotherapy) }\end{array}$ & $60 / 28$ & $50 / 28$ & Cisplatin \\
\hline Peeters [21] & 2006 & Prostate & 641 & 146 & Anal wall & $\begin{array}{l}\text { RTOG/EORTC } \\
\text { plus } 5 \text { specified } \\
\text { symptoms }\end{array}$ & $\begin{array}{l}\text { 3D ( } 41 \text { pts. } \\
\text { had IMRT } \\
\text { boost) }\end{array}$ & $68 / 34$ or $78 / 39$ & NS & no \\
\hline Peeters [28] & 2006 & Prostate & 368 & 32 & Anal wall & $\begin{array}{l}\text { Incontinence } \\
\text { (no specific } \\
\text { questionnaire) }\end{array}$ & $\begin{array}{l}3 \mathrm{D} \text { ( } 22 \mathrm{pts} . \\
\text { had boost) }\end{array}$ & $68 / 34$ or $78 / 39$ & NS & no \\
\hline Poorvu [16] & 2013 & $\begin{array}{l}\text { Cervix or } \\
\text { Endometrium } \\
\text { (+ PA nodes) }\end{array}$ & 46 & 3 & $\begin{array}{l}\text { peritoneal } \\
\text { cavity, small } \\
\text { bowel, } \\
\text { duodenal } \\
\text { segments }\end{array}$ & CTCAE v4.0 & IMRT & $\begin{array}{l}\text { 45/25 (22pts); } \\
\text { PAN boost 50- } \\
65 \text { (33pts) }\end{array}$ & $\begin{array}{l}45 / 25 \& \\
\text { PAN boost } \\
50-65 \text { (33 } \\
\text { pts) }\end{array}$ & $\begin{array}{l}24 \text { received } \\
\text { cisplatin }\end{array}$ \\
\hline $\begin{array}{l}\text { Smeenk } \\
{[26]}\end{array}$ & 2012 & Prostate & 48 & 21 & $\begin{array}{l}\text { Anal } \\
\text { sphincter } \\
\text { muscles }\end{array}$ & $\begin{array}{l}\text { Presence of } \\
\text { frequency, } \\
\text { urgency and } \\
\text { incontinence }\end{array}$ & $\begin{array}{l}3 \mathrm{D}(n=43 \\
\text { IMRT }(n=5)\end{array}$ & $\begin{array}{l}67.5 / 27 \text { or } 70 / \\
28\end{array}$ & NS & no \\
\hline $\begin{array}{l}\text { Smeenk } \\
{[22]}\end{array}$ & 2012 & Prostate & 36 & 23 & Anal wall & $\begin{array}{l}\text { Late RILIT score: } \\
\text { urgency, } \\
\text { incontinence, } \\
\text { frequency }\end{array}$ & $3 \mathrm{D}$ & $\begin{array}{l}67.5 / 27 \text { or } 70 / \\
28\end{array}$ & NS & no \\
\hline Taussky [37] & 2003 & Prostate & 73 & unclear & anal canal & $\begin{array}{l}\text { UCLA, FACT-P } \\
\text { and EORTC } \\
\text { QLQ-PR25 }\end{array}$ & $3 \mathrm{D}$ & $66.6-72 / 37-40$ & NS & no \\
\hline Thor [29] & 2015 & Prostate & 212 & $\begin{array}{l}\text { Symptom } \\
\text { specific }\end{array}$ & $\begin{array}{l}\text { Anal } \\
\text { sphincter }\end{array}$ & $\begin{array}{l}\text { Own } \\
\text { questionnaire } \\
\text { with } 19 \\
\text { descriptors for } 4 \\
\text { symptoms }\end{array}$ & $3 \mathrm{D}$ & 70-78Gy & NS & no \\
\hline Verma [30] & 2014 & $\begin{array}{l}\text { Cervix \& } \\
\text { Endometrium }\end{array}$ & 105 & 9 & duodenum & $\begin{array}{l}\text { RTOG and } \\
\text { endoscopic } \\
\text { findings }\end{array}$ & IMRT & $\begin{array}{l}45-50 \text { (60-66Gy } \\
\text { boost) }\end{array}$ & $\begin{array}{l}45-50 \\
(60-66 \\
\text { boost) }\end{array}$ & $\begin{array}{l}58 \text { pts. } \\
\text { platinum } \\
\text { agents }\end{array}$ \\
\hline $\begin{array}{l}\text { Vordermark } \\
{[23]}\end{array}$ & 2003 & $\begin{array}{l}\text { Prostate or } \\
\text { prostatic bed }\end{array}$ & 44 & $\begin{array}{l}14 \% \text { severe } \\
\text { incontinence }\end{array}$ & anal canal & $\begin{array}{l}10 \text { question } \\
\text { continence } \\
\text { questionnaire }\end{array}$ & $3 \mathrm{D}$ & $58-72 / 29-36$ & NS & No \\
\hline Yeoh [24] & 2016 & Prostate & 106 & $72 \%$ & Anal wall & $\begin{array}{l}\text { LENT-SOMA } \\
\text { total score }\end{array}$ & $3 \mathrm{D}$ & $66-74.4 / 33-4$ & NS & no \\
\hline
\end{tabular}

Abbreviations: Pts Patients, OAR Organs at risk, RT Radiotherapy, Gy Gray, \# Fraction, NS Not stated, CTCAE Common terminology criteria for adverse events, RTOG Radiation therapy oncology group, LENT-SOMA Late Effects of Normal Tissue - Subjective Objective Management Analytical, RILIT Radiation induced late intestinal toxicity, EORTC European Organisation for Research and Treatment of Cancer, IG-IMRT Image-guided intensity modulated radiotherapy, IMRT Intensity modulated radiotherapy, VMAT Volumetric modulated arc therapy, PA nodes Para-aortic nodes, pts Patients

constraints-V15 $<250 \mathrm{cc}, \mathrm{V} 30<100 \mathrm{cc}$ and $\mathrm{V} 40<90 \mathrm{cc}$ grade 3 toxicity could reduce from 26.7 to $5.4 \%$.

For the sigmoid colon Fonteyne et al. [17] found in 241 prostate patients that sigmoid V40 was associated with grade 1 diarrhoea and blood loss; they recommended $\mathrm{V} 40<10 \%$ and $\mathrm{V} 30<16 \%$ to avoid grade $1-2$ diarrhoea. Mouttet-Aldouard et al. [6] also found sigmoid V30-40Gy to be significantly correlated $(p<0.006)$ with "digestive toxicity" although no specific cut-offs were defined.

\section{Anal canal}

15 of 16 studies had positive findings relating dose-volume parameters and Normal Tissue Complication Probability (NTCP) models of the anal canal/ sphincter to late toxicity. Most defined the anal canal as the distal $3 \mathrm{~cm}$ of rectum.

\section{Dmean}

5 studies [18-22] found Dmean anal canal or anal sphincter region to be most predictive of toxicity, 4 of 
Table 3 Whole bowel and small bowel studies - significant findings and quality assessment

\begin{tabular}{|c|c|c|c|c|c|c|c|}
\hline \multirow[b]{2}{*}{ Author } & \multirow[b]{2}{*}{ OAR studied } & \multirow[b]{2}{*}{ OAR defined } & \multirow[b]{2}{*}{ Toxicity definition } & \multirow[b]{2}{*}{$\begin{array}{l}\text { Pts with } \\
\text { toxicity }\end{array}$} & \multirow[b]{2}{*}{ Significant findings } & \multicolumn{2}{|c|}{ Quality Assessment } \\
\hline & & & & & & $\begin{array}{l}\text { Statistical } \\
\text { criteria } \\
\text { met (1-7) }\end{array}$ & $\begin{array}{l}\text { Endpoint } \\
\text { criteria } \\
\text { met }(1-3)\end{array}$ \\
\hline Deville [7] & Intestinal cavity & Intestinal cavity below L4-5 & RTOG Gr $\geq 1$ & $\begin{array}{l}5 / 36 \\
(14 \%)\end{array}$ & $\begin{array}{l}\text { Toxicity associated with } \\
\text { total volume \& V20. No } \\
\text { constraints specified. }\end{array}$ & $\begin{array}{l}1,7 \\
\text { (n/a: 2-6) }\end{array}$ & None \\
\hline $\begin{array}{l}\text { Mouttet- } \\
\text { Audouard } \\
{[6]}\end{array}$ & $\begin{array}{l}\text { "Small bowel" } \\
\text { [outlined as } \\
\text { abdominal } \\
\text { cavity hence } \\
\text { included in } \\
\text { this section] }\end{array}$ & $\begin{array}{l}\text { Entire abdominal cavity } \\
\text { including all possible organ } \\
\text { locations to iliac crests or } \\
\text { D12/L1 }\end{array}$ & $\begin{array}{l}\text { CTCAE v4.0 Gr1-3 - } \\
\text { diarrhoea or "whole } \\
\text { digestive toxicity" } \\
\text { (diarrhoea, gastritis, } \\
\text { bleeding, pain, } \\
\text { incontinence) }\end{array}$ & $\begin{array}{l}8 / 37 \\
(21.6 \%) \\
17 / 37 \\
(46 \%)\end{array}$ & $\begin{array}{l}\text { Larger volumes of bowel } \\
\text { receiving 10-30Gy } \\
\text { associated with diarrhoea \& } \\
\text { whole digestive toxicity. (No } \\
\text { constraints specified) } \\
\text { "Whole digestive toxicity" } \\
\text { associated with many } \\
\text { parameters including D20\%- } \\
\text { D95\%. }\end{array}$ & $\begin{array}{l}1,7 \\
(n / a 2-6)\end{array}$ & 2 \\
\hline Green [36] & Intestinal cavity & Not stated & CTCAE v4.0 & $9(12 \%)$ & $\begin{array}{l}\text { No dose-volume relation- } \\
\text { ship found. }\end{array}$ & $\begin{array}{l}1(\mathrm{n} / \mathrm{a} 2- \\
6)\end{array}$ & 2 \\
\hline $\begin{array}{l}\text { Deville } \\
{[31]}\end{array}$ & Intestinal cavity & $\begin{array}{l}\text { Large \& small bowel below } \\
\text { L4-5 }\end{array}$ & RTOG Gr $\geq 2$ & $2 / 30(6 \%)$ & $\begin{array}{l}\text { No dose-volume relation- } \\
\text { ship found }\end{array}$ & $\begin{array}{l}1,7 \\
(\mathrm{n} / \mathrm{a} 2-6)\end{array}$ & None \\
\hline $\begin{array}{l}\text { Isohashi } \\
{[10]}\end{array}$ & $\begin{array}{l}\text { Peritoneal } \\
\text { cavity }\end{array}$ & $\begin{array}{l}\text { Volume surrounding small } \\
\text { bowel loops to edge of } \\
\text { peritoneum excluding } \\
\text { bladder \& rectum }\end{array}$ & RTOG/EORTC Gr $\geq 2$ & $\begin{array}{l}16 / 97 \\
(16.5 \%)\end{array}$ & $\begin{array}{l}\text { No dose-volume relation- } \\
\text { ship found }\end{array}$ & $\begin{array}{l}1,7 \\
(\mathrm{n} / \mathrm{a} 2-6)\end{array}$ & 2 \\
\hline $\begin{array}{l}\text { Poorvu } \\
{[16]}\end{array}$ & $\begin{array}{l}\text { 1. Peritoneum } \\
\text { 2. Peritoneum } \\
+ \text { Colon }\end{array}$ & $\begin{array}{l}\text { 1. Possible location of small } \\
\text { bowel excluding solid organs } \\
\text { \& retroperitoneal structures. } \\
\text { 2. Peritoneum (as above) plus } \\
\text { asc \& desc colon }\end{array}$ & CTCAE v4.0 Gr $>3$ & $\begin{array}{l}3 / 46 \\
(6.5 \%)\end{array}$ & $\begin{array}{l}\text { No dose-volume relation- } \\
\text { ship found }\end{array}$ & $\begin{array}{l}1,7 \\
(\mathrm{n} / \mathrm{a} 2-6)\end{array}$ & 2 \\
\hline $\begin{array}{l}\text { Guerrero- } \\
\text { Urbano [8] }\end{array}$ & Bowel loops & $\begin{array}{l}\text { Loops from recto-sigmoid } \\
\text { junction to } 2 \mathrm{~cm} \text { above PTV }\end{array}$ & $\begin{array}{l}\text { RTOG Gr } \geq 2 \\
\text { diarrhoea; LENT- } \\
\text { SOMA consistency \& } \\
\text { frequency- worst } \\
\text { grade }\end{array}$ & $\begin{array}{l}21 / 79 \\
(26 \%) \\
\text { RTOG } \\
\text { diarrhoea; } \\
\geq \text { gr2 6/79 } \\
(7.6 \%)\end{array}$ & $\begin{array}{l}\text { V40, V45, V60 and bowel } \\
\text { volume of }>450 \mathrm{cc} \text { had } \\
\text { both higher RTOG \& } \\
\text { LENTSOMA diarrhoea. } \\
\text { Constraints suggested: V40 } \\
<124 \mathrm{cc} \text {, V45 < } 71 \mathrm{cc} \text {, V60< } \\
0.5 \mathrm{cc} \text { for RTOG<gr } 2\end{array}$ & $\begin{array}{l}1,7 \\
(\mathrm{n} / \mathrm{a} 2-3)\end{array}$ & 1 \\
\hline $\begin{array}{l}\text { McDonald } \\
{[9]}\end{array}$ & Bowel loops & $\begin{array}{l}\text { Loops from recto-sigmoid } \\
\text { junction to } 2 \mathrm{~cm} \text { above PTV }\end{array}$ & RTOG Gr $\geq 1$ & $\begin{array}{l}7 / 47 \\
(14.9 \%) \\
\operatorname{gr} 1 ; 3 / 47 \\
(6.4 \%) \mathrm{gr} 2\end{array}$ & 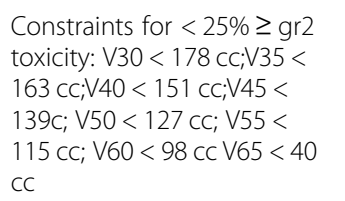 & $\begin{array}{l}1,4,7 \\
(\mathrm{n} / \mathrm{a} 2-3)\end{array}$ & 2 \\
\hline $\begin{array}{l}\text { Chopra } \\
{[11]}\end{array}$ & Small bowel & $\begin{array}{l}2 \mathrm{~cm} \text { above target, individual } \\
\text { small bowel loops (unclear } \\
\text { how differentiated from large } \\
\text { bowel) }\end{array}$ & CTCAE v3.0 Gr3+ & $\begin{array}{l}9 / 71 \\
(12.6 \%)\end{array}$ & $\begin{array}{l}\text { V15 associated with } \geq g r 3 \\
\text { toxicity. Recommend V15< } \\
275 \mathrm{cc} \text { V } 30<190 \mathrm{cc}, \mathrm{V} 40< \\
150 \mathrm{cc} \text { reduces Gr3 toxicity } \\
\text { from } 23.6 \text { to } 5.6 \% \text {. }\end{array}$ & $\begin{array}{l}1,4,6 \\
(\mathrm{n} / \mathrm{a} 2,3)\end{array}$ & 2 \\
\hline $\begin{array}{l}\text { Isohashi } \\
{[10]}\end{array}$ & Small bowel & $\begin{array}{l}\text { Bowel loops remaining after } \\
\text { exclusion of large bowel } \\
\text { loops }\end{array}$ & RTOG/EORTC Gr $\geq 2$ & $\begin{array}{l}16 / 97 \\
(16.5 \%)\end{array}$ & $\begin{array}{l}\text { V } 40 \text { best predictor of late } \\
\text { toxicity; Recommend } V 40< \\
340 \mathrm{ml} \text { to reduce toxicity } \\
\text { from } 46.2 \text { to } 8.7 \%\end{array}$ & $\begin{array}{l}1,4,6,7 \\
(\mathrm{n} / \mathrm{a} 2,3)\end{array}$ & 2 \\
\hline Lind [12] & Small bowel & $\begin{array}{l}\text { All visible small bowel in } \\
\text { small pelvic cavity to caudal } \\
\text { part of sacroiliac joints }\end{array}$ & $\begin{array}{l}\text { Defecation into } \\
\text { clothing without } \\
\text { warning }>1 \text { in last } 6 \\
\text { months }\end{array}$ & $\begin{array}{l}63 / 519 \\
(12.1 \%)\end{array}$ & $\begin{array}{l}\text { Mean dose }>50 \text { Gy to small } \\
\text { bowel or sigmoid or anal } \\
\text { sphincter region associated } \\
\text { with symptom (findings for } \\
\text { individual organs not } \\
\text { clarified) }\end{array}$ & $\begin{array}{l}1,7(n / a \\
2,3)\end{array}$ & $1,2,3$ \\
\hline $\begin{array}{l}\text { Adkison } \\
{[34]}\end{array}$ & Small bowel & Not clearly defined & $\begin{array}{l}\text { CTCAE v3.0 Gr1 and } \\
\text { Gr2 }\end{array}$ & $\begin{array}{l}\text { Gr1 16/53 } \\
(30 \%) ; G r 2 \\
4 / 53(8 \%)\end{array}$ & $\begin{array}{l}\text { No dose-volume relation- } \\
\text { ship with V30-V60 small } \\
\text { bowel }\end{array}$ & $\begin{array}{l}1 \\
(n / a 2-6)\end{array}$ & None \\
\hline Fokdal [14] & Small bowel & $\begin{array}{l}\text { Opacified \& unopacified small } \\
\text { intestine loops (outer contour }\end{array}$ & LENT-SOMA G1-4 & $\begin{array}{l}\text { Symptom } \\
\text { specific }\end{array}$ & $\begin{array}{l}\text { No dose-volume relation- } \\
\text { ship found }\end{array}$ & $\begin{array}{l}1,7 \\
(\mathrm{n} / \mathrm{a} 2-6)\end{array}$ & $1,2,3$ \\
\hline
\end{tabular}


Table 3 Whole bowel and small bowel studies - significant findings and quality assessment (Continued)

\begin{tabular}{|c|c|c|c|c|c|c|c|}
\hline \multirow[b]{2}{*}{ Author } & \multirow[b]{2}{*}{ OAR studied } & \multirow[b]{2}{*}{ OAR defined } & \multirow[b]{2}{*}{ Toxicity definition } & \multirow[b]{2}{*}{$\begin{array}{l}\text { Pts with } \\
\text { toxicity }\end{array}$} & \multirow[b]{2}{*}{ Significant findings } & \multicolumn{2}{|c|}{ Quality Assessment } \\
\hline & & & & & & $\begin{array}{l}\text { Statistical } \\
\text { criteria } \\
\text { met }(1-7)\end{array}$ & $\begin{array}{l}\text { Endpoint } \\
\text { criteria } \\
\text { met }(1-3)\end{array}$ \\
\hline & & $\begin{array}{l}\& \text { contents) from 1st slice to } \\
\text { minor pelvis }\end{array}$ & & & & & \\
\hline $\begin{array}{l}\text { Fonteyne } \\
{[17]}\end{array}$ & Small bowel & Not clearly defined & $\begin{array}{l}\text { RTOG and "RILIT" Gr1 } \\
\& \text { Gr2 }\end{array}$ & $\begin{array}{l}\text { Gr1 } 112 / \\
241(46 \%) \\
\text { Gr2 32/ } \\
241(13 \%)\end{array}$ & $\begin{array}{l}\text { No dose-volume relation- } \\
\text { ship found }\end{array}$ & $\begin{array}{l}1, \\
(n / a 2-6)\end{array}$ & 1,2 \\
\hline $\begin{array}{l}\text { Poorvu } \\
{[16]}\end{array}$ & Small bowel & $\begin{array}{l}\text { Opacified \& non-opacified } \\
\text { small bowel loops }\end{array}$ & CTCAE v4.0 Gr3+ & $\begin{array}{l}3 / 46 \\
(6.5 \%)\end{array}$ & $\begin{array}{l}\text { No dose-volume relation- } \\
\text { ship found }\end{array}$ & $\begin{array}{l}1,7 \\
(n / a 2-6)\end{array}$ & 2 \\
\hline $\begin{array}{l}\text { Huang } \\
{[15]}\end{array}$ & Duodenum & $\begin{array}{l}\text { Duodenal bulb to ligament of } \\
\text { Treitz }\end{array}$ & CTCAE $\vee 4.0 \mathrm{Gr} \geq 3$ & $\begin{array}{l}8 / 46 \\
(17.4 \%)\end{array}$ & $\begin{array}{l}\text { With a V25 }>45 \% \text { toxicity } \\
\text { rates increase from } 8 \text { to } \\
48 \%\end{array}$ & $\begin{array}{l}1,4,6,7 \\
(\mathrm{n} / \mathrm{a} 2,3)\end{array}$ & 2 \\
\hline Kelly [13] & Duodenum & $\begin{array}{l}\text { Gastric pylorus until end of } \\
\text { duodenum } 3 \mathrm{~cm} \text { past midline }\end{array}$ & CTCAE v4.0 Gr $\geq 2$ & $\begin{array}{l}20 / 106 \\
(18.9 \%)\end{array}$ & $\begin{array}{l}\text { With a V } 55>1 \text { cc toxicity } \\
\text { rates increase from } 9 \text { to } \\
47 \%\end{array}$ & $\begin{array}{l}1,4,6,7 \\
(\mathrm{n} / \mathrm{a} 2,3)\end{array}$ & 2 \\
\hline Verma [30] & Duodenum & $\begin{array}{l}\text { From gastric outlet through } \\
\text { transverse portion of } \\
\text { duodenum (ascending } \\
\text { portion excluded) }\end{array}$ & RTOG, all grades & $\begin{array}{l}9 / 105 \\
(8.6 \%)\end{array}$ & $\begin{array}{l}\text { With a } V 55>15 \mathrm{cc} \text { toxicity } \\
\text { rates increase from } 7.4 \text { to } \\
48.6 \%\end{array}$ & $\begin{array}{l}1,4,6,7 \\
(\mathrm{n} / \mathrm{a} 2,3)\end{array}$ & 2 \\
\hline $\begin{array}{l}\text { Poorvu } \\
{[16]}\end{array}$ & $\begin{array}{l}\text { Duodenal } \\
\text { segments }\end{array}$ & $\begin{array}{l}\text { D1 segment: bulblike shape \& } \\
\text { origin beyond gastric pylorus. } \\
\text { Transitions between } 2 \text { nd \& } \\
\text { 3rd segments was lateral } \\
\text { border of IVC; Between 3rd \& } \\
\text { 4th was medial border of } \\
\text { aorta }\end{array}$ & CTCAE v4.0 Gr $\geq 3$ & $\begin{array}{l}3 / 46 \\
(6.5 \%)\end{array}$ & $\begin{array}{l}\text { No dose-volume relation- } \\
\text { ship found with duodenum }\end{array}$ & $\begin{array}{l}1,7 \\
(\mathrm{n} / \mathrm{a} 2-6)\end{array}$ & 2 \\
\hline
\end{tabular}

Abbreviations: Pts Patients, OAR Organs at risk, RT Radiotherapy, Gr Grade, CTCAE Common terminology criteria for adverse events, RTOG Radiation therapy oncology group, LENT-SOMA Late Effects of Normal Tissue - Subjective Objective Management Analytical, RILIT Radiation induced late intestinal toxicity, EORTC European Organisation for Research and Treatment of Cancer, Vx Volume receiving $x$ Gy, AUC Area under curve

faecal incontinence and 1 of faecal urgency, as summarised in Table 5. There was relative consistency in the recommended Dmean constraints between 40-47Gy, despite the OARs being defined slightly differently.

\section{Other dose volume histogram (DVH) and dose-surface histogram (DSH) parameters}

Many other DVH parameters of the anal canal were found to be important, including Dmin [23], Dmax, Dmedian [14], V40 [24], V65 [21] and V90\% dose [25]; these were all in individual findings with little corroboration between studies. Vordermark et al. also found that the treatment field border was important, with those with a lower border $2 \mathrm{~mm}$ below ischial tuberosities more likely to have severe incontinence compared with $5 \mathrm{~mm}$ above the ischial tuberosities.

Buettner et al. [20] also examined incontinence using dose surface maps (DSM) for the anal canal. They found the mean dose to the anal surface and the lateral extent of the DSM to be most correlated with subjective sphincter toxicity. They recommend 45Gy for surface-based mean-dose to the anal canal to reduce toxicity.

\section{Anal sphincter muscles}

Smeenk et al. [26] related dose to individual sphincter muscles to urgency, frequency and incontinence. To reduce urgency and incontinence to below 5\% they recommended a mean dose $<30$ Gy to internal anal sphincter, $<10 \mathrm{~Gy}$ to the external sphincter, $<50 \mathrm{~Gy}$ to puborectalis and $<40 G y$ to the levator ani muscles.

\section{Normal tissue complication probability (NTCP) modeling}

Four studies detailed NTCP models for the anal canal [20, 27-29], three of fitting data to a Lyman-Kutcher-Burman (LKB) model. Buettner et al. identified mean-dose anal canal parameters related to grade 2 RTOG toxicity, and Peeters et al. looked at anal wall parameters in relation to faecal incontinence, as detailed in Table 4. Peeters et al. further modified their model to incorporate a previous history of abdominal surgery and found this improved the model fit, suggesting a decreased radiation tolerance for patients with this risk factor. Thor et al. [29] proposed LKB models for pain, mucus and faecal leakage, although their findings are difficult to use practically as within their study they use data from two different centres, where each toxicity is defined differently between centres. Mavroidis et al. 
Table 4 Large Bowel studies - details and quality assessment

\begin{tabular}{|c|c|c|c|c|c|c|c|}
\hline \multirow[b]{2}{*}{ Author } & \multirow[b]{2}{*}{$\begin{array}{l}\text { OAR } \\
\text { studied }\end{array}$} & \multirow[b]{2}{*}{ OAR defined } & \multirow[b]{2}{*}{$\begin{array}{l}\text { Toxicity } \\
\text { definition }\end{array}$} & \multirow[b]{2}{*}{$\begin{array}{l}\text { Pts with } \\
\text { toxicity }\end{array}$} & \multirow[b]{2}{*}{ Significant findings } & \multicolumn{2}{|c|}{ Quality Assessment } \\
\hline & & & & & & $\begin{array}{l}\text { Statistical } \\
\text { considerations } \\
\text { met }(1-7)\end{array}$ & $\begin{array}{l}\text { Endpoint } \\
\text { considerations } \\
\text { met }(1-3)\end{array}$ \\
\hline Chopra [11] & $\begin{array}{l}\text { Large } \\
\text { bowel }\end{array}$ & $\begin{array}{l}2 \mathrm{~cm} \text { above target, } \\
\text { individual loops of large } \\
\text { bowel (unclear how } \\
\text { differentiated from small } \\
\text { bowel) }\end{array}$ & $\begin{array}{l}\text { CTCAE v } 3.0 \\
\text { Gr } \geq 3\end{array}$ & 9/71 (12.6\%) & $\begin{array}{l}\text { V15 associated with } \geq \mathrm{gr} 3 \\
\text { toxicity. } \\
\text { Constraints: } \mathrm{V} 15<250 \mathrm{cc} \text {, } \\
\text { V30<100 cc, V } 40<90 \mathrm{cc} \text { to } \\
\text { reduce toxicity from } 26.7 \text { to } \\
5.4 \%\end{array}$ & $\begin{array}{l}1,4,6 \\
(n / a 2,3)\end{array}$ & 2 \\
\hline $\begin{array}{l}\text { Isohashi } \\
{[10]}\end{array}$ & $\begin{array}{l}\text { Large } \\
\text { bowel }\end{array}$ & $\begin{array}{l}\text { Single loop continuing } \\
\text { from end of sigmoid to } \\
\text { ascending colon }\end{array}$ & $\begin{array}{l}\text { RTOG/EORTC, } \\
\text { Gr } \geq 2\end{array}$ & 16/97 (16.5\%) & $\begin{array}{l}\text { No constraint found for large } \\
\text { bowel }\end{array}$ & $\begin{array}{l}1,7 \\
(n / a) 2-6)\end{array}$ & 2 \\
\hline $\begin{array}{l}\text { Fonteyne } \\
{[17]}\end{array}$ & $\begin{array}{l}\text { Sigmoid } \\
\text { colon }\end{array}$ & $\begin{array}{l}\text { Where rectum sweeps } \\
\text { anteriorly to one slice } \\
\text { above aortic bifurcation }\end{array}$ & $\begin{array}{l}\text { RTOG and } \\
\text { "RILIT" Gr } 1 \text { and } \\
2\end{array}$ & $\begin{array}{l}\text { Gr } 1112 / 241 \\
(46 \%), \text { Gr } 2 \\
32 / 241(13 \%)\end{array}$ & $\begin{array}{l}\text { V40 associated with gr1 } \\
\text { diarrhoea \& blood loss. } \\
\text { Constraints: V40 < 10\%, V30 } \\
<16 \% \text { to avoid gr1-2 } \\
\text { diarrhoea }\end{array}$ & $\begin{array}{l}1,7 \\
(\mathrm{n} / \mathrm{a} 3)\end{array}$ & 1,2 \\
\hline $\begin{array}{l}\text { Mouttet- } \\
\text { Audouard } \\
{[6]}\end{array}$ & $\begin{array}{l}\text { Sigmoid } \\
\text { colon }\end{array}$ & $\begin{array}{l}\text { Anterior curvature of } \\
\text { sigmoid colon to } \\
\text { anterior abdominal wall }\end{array}$ & $\begin{array}{l}\text { CTCAE v4.0 } \\
\text { Gr1-3 diarrhoea } \\
\text { and "whole } \\
\text { digestive } \\
\text { toxicity" }\end{array}$ & $\begin{array}{l}8 / 37(21.6 \%) \\
\text { diarrhoea; } 17 / \\
37 \\
(46 \%) \text { (whole } \\
\text { tox) }\end{array}$ & $\begin{array}{l}\text { 'Whole late digestive toxicity' } \\
\text { associated with V30-40. No } \\
\text { specific constraints. }\end{array}$ & $\begin{array}{l}1,7 \\
(\mathrm{n} / \mathrm{a} 2-6)\end{array}$ & 1,2 \\
\hline Lind [12] & $\begin{array}{l}\text { Sigmoid } \\
\text { colon }\end{array}$ & $\begin{array}{l}\text { From where rectum } \\
\text { deviates from its mid- } \\
\text { position to where it } \\
\text { turns cranially in left } \\
\text { abdomen connecting to } \\
\text { colon descendens }\end{array}$ & $\begin{array}{l}\text { Defecation into } \\
\text { clothing } \\
\text { without } \\
\text { warning }>1 \text { in } \\
\text { last } 6 \text { months }\end{array}$ & $\begin{array}{l}63 / 519 \\
(12.1 \%)\end{array}$ & $\begin{array}{l}\text { Mean dose>50Gy to small } \\
\text { bowel or sigmoid or anal } \\
\text { sphincter region associated } \\
\text { with symptom (findings for } \\
\text { individual organs not } \\
\text { clarified) }\end{array}$ & $\begin{array}{l}1,7 \\
(\mathrm{n} / \mathrm{a} 2-6)\end{array}$ & $1,2,3$ \\
\hline $\begin{array}{l}\text { al-Abany } \\
{[18]}\end{array}$ & $\begin{array}{l}\text { Anal } \\
\text { sphincter } \\
\text { region }\end{array}$ & $\begin{array}{l}\text { Caudal } 3 \mathrm{~cm} \text { of the } \\
\text { rectum from anal verge } \\
\text { (including filling) }\end{array}$ & $\begin{array}{l}\text { Own } \\
\text { questionnaire; } \\
\text { Faecal leakage } \\
>2 X / \text { week }\end{array}$ & $\begin{array}{l}9 / 65(13.8 \%) \\
\text { faecal leakage }\end{array}$ & $\begin{array}{l}\text { Increased risk with mean } \\
\text { dose of } 45-55 G y \text {. } \\
\text { Constraints: V } 35<60 \% \text {, V40 } \\
<40 \% \text { associated with no } \\
\text { risk of faecal leakage. }\end{array}$ & $\begin{array}{l}1,7 \\
(n / a) 2,3)\end{array}$ & $1,2,3$ \\
\hline $\begin{array}{l}\text { Alsadius } \\
\text { [19] }\end{array}$ & $\begin{array}{l}\text { Anal } \\
\text { sphincter } \\
\text { region }\end{array}$ & $\begin{array}{l}\text { Caudal part of large } \\
\text { bowel, from end of } \\
\text { rectal ampulla where } \\
\text { bowel no longer had } \\
\text { visible content or air. }\end{array}$ & $\begin{array}{l}\text { Own } \\
\text { questionnaire; } \\
\text { Faecal leakage } \\
\text { >once per } \\
\text { month }\end{array}$ & $\begin{array}{l}51 / 403 \\
(12.7 \%) \text { faecal } \\
\text { leakage }\end{array}$ & $\begin{array}{l}\text { Dmean< }<40 \text { Gy reduces risk } \\
\text { from } 17 \text { to } 4 \% \text {. }\end{array}$ & $\begin{array}{l}1,2,4,7 \\
\text { (n/a } 3)\end{array}$ & $1,2,3$ \\
\hline Fokdal [14] & $\begin{array}{l}\text { Anal } \\
\text { canal }\end{array}$ & $\begin{array}{l}\text { Outer contour of the } \\
\text { structure extending from } \\
\text { anal verge } 2 \mathrm{~cm} \text { cranially }\end{array}$ & $\begin{array}{l}\text { LENT SOMA } \\
\text { score }\end{array}$ & $\begin{array}{l}\text { Urge: } 27 / 71 \\
(38 \%) ; \\
\text { Incontinence: } \\
21 / 71(30 \%)\end{array}$ & $\begin{array}{l}\text { Urgency related to Dmed> } \\
33.8: \text { increases toxicity } 31 \text { to } \\
47 \% \\
\text { Incontinence related to } \\
\text { Dmax> } 53.8 \text { increases } 14 \text { to } \\
44 \%\end{array}$ & $\begin{array}{l}1,2,4,5,7 \\
(\mathrm{n} / \mathrm{a} 3)\end{array}$ & $1,2,3$ \\
\hline $\begin{array}{l}\text { Vordermark } \\
{[23]}\end{array}$ & $\begin{array}{l}\text { Anal } \\
\text { canal }\end{array}$ & $\begin{array}{l}\text { Anal verge to the } \\
\text { section below visible } \\
\text { rectal lumen, } \\
\text { corresponding to the } \\
\text { upper border of the } \\
\text { levator ani muscle }\end{array}$ & $\begin{array}{l}\text { "Solid soiling" } \\
\text { (Severe } \\
\text { incontinence) } \\
\text { Own } \\
\text { continence } \\
\text { questionnaire }\end{array}$ & $6 / 44(14 \%)$ & $\begin{array}{l}\text { Severe incontinence } \\
\text { - associated with Dmin } \\
\text { ( } 23.1 \mathrm{~Gy}) \\
\text { - related to portals } \\
\text { extending } 2 \mathrm{~mm} \text { below } \\
\text { ischial tuberosities } \\
\text { (compared with } 5 \mathrm{~mm} \\
\text { above) }\end{array}$ & $\begin{array}{l}1,7 \\
(n / a) 2-3)\end{array}$ & $1,2,3$ \\
\hline Koper [25] & $\begin{array}{l}\text { Anal } \\
\text { canal }\end{array}$ & $\begin{array}{l}\text { Caudal } 3 \mathrm{~cm} \text { of the } \\
\text { intestine }\end{array}$ & $\begin{array}{l}\text { RTOG gr1 + 2; } \\
\text { Plus symptom } \\
\text { questionnaire. }\end{array}$ & $\begin{array}{l}141 / 248 \\
(57 \%)\end{array}$ & $\begin{array}{l}\mathrm{D} 90 \%(=54.9 \mathrm{~Gy}) \text { to } \\
\text { associated with } \geq \text { gr1 rectal } \\
\text { toxicity }\end{array}$ & $\begin{array}{l}1,7 \\
(\mathrm{n} / \mathrm{a} 2-6)\end{array}$ & 2,3 \\
\hline Taussky [37] & $\begin{array}{l}\text { Anal } \\
\text { canal }\end{array}$ & $\begin{array}{l}\text { Most distal } 2-3 \mathrm{~cm} \text { of } \\
\text { rectum }\end{array}$ & $\begin{array}{l}10 \text { questions } \\
\text { from UCLA-PCI, } \\
\text { FACT-P \& } \\
\text { EORTC QLQ } \\
\text {-PR25 }\end{array}$ & Unclear & $\begin{array}{l}\text { no relation with anal canal } \\
\text { DVH found }\end{array}$ & $\begin{array}{l}7 \\
\text { (N/a: 2-3) }\end{array}$ & 3 \\
\hline $\begin{array}{l}\text { Buettner } \\
{[20]}\end{array}$ & $\begin{array}{l}\text { Anal } \\
\text { canal }\end{array}$ & Caudal $3 \mathrm{~cm}$ of rectum & $\begin{array}{l}\text { Common } \\
\text { grading }\end{array}$ & $\begin{array}{l}57 / 388 \\
(14.7 \%)\end{array}$ & $\begin{array}{l}\text { DSH data: Toxicity correlated } \\
\text { with dose to anal surface: }\end{array}$ & $\begin{array}{l}1,3,6,7 \\
(\mathrm{n} / \mathrm{a} 2)\end{array}$ & $1,2,3$ \\
\hline
\end{tabular}


Table 4 Large Bowel studies - details and quality assessment (Continued)

\begin{tabular}{|c|c|c|c|c|c|c|c|}
\hline \multirow[b]{2}{*}{ Author } & \multirow[b]{2}{*}{$\begin{array}{l}\text { OAR } \\
\text { studied }\end{array}$} & \multirow[b]{2}{*}{ OAR defined } & \multirow[b]{2}{*}{$\begin{array}{l}\text { Toxicity } \\
\text { definition }\end{array}$} & \multirow[b]{2}{*}{$\begin{array}{l}\text { Pts with } \\
\text { toxicity }\end{array}$} & \multirow[b]{2}{*}{ Significant findings } & \multicolumn{2}{|c|}{ Quality Assessment } \\
\hline & & & & & & $\begin{array}{l}\text { Statistical } \\
\text { considerations } \\
\text { met }(1-7)\end{array}$ & $\begin{array}{l}\text { Endpoint } \\
\text { considerations } \\
\text { met }(1-3)\end{array}$ \\
\hline & & & $\begin{array}{l}\text { scheme; } \\
\text { subjective } \\
\text { sphincter } \\
\text { control at } \\
\text { highest grade }\end{array}$ & & $\begin{array}{l}\text { lateral extent 53Gy > 56\%. } \\
\text { DVH data: Dmean 47Gy to } \\
\text { anal sphincter volume } \\
\text { correlated with sphincter } \\
\text { toxicity. Constraints: } \\
\text { Dmean<30Gy.NTCP } \\
\text { modeling to LKB model } \\
\text { TD50 }=120, \mathrm{~m}=0.42 \text {. }\end{array}$ & & \\
\hline Peeters [21] & Anal wall & $\begin{array}{l}\text { Wall of caudal } 3 \mathrm{~cm} \text { of } \\
\text { anorectum (method } \\
\text { described) }\end{array}$ & $\begin{array}{l}\text { RTOG/EORTC } \geq \\
\text { gr } 2 \text { and } \geq \text { gr } 3 \\
\text { Plus } \\
\text { incontinence } \\
\text { pad use }>2 x / w k\end{array}$ & $\begin{array}{l}\geq \operatorname{gr} 2165 / 641 \\
(25.7 \%) \geq \text { gr } 3 \\
27 / 6414.2 \%\end{array}$ & $\begin{array}{l}\text { Dmean increase from 19Gy } \\
\text { to } 52 \mathrm{~Gy} \text { increased gr2 } \\
\text { toxicity: } 16 \text { to } 31 \% \text {. } \\
\text { V65 \& Dmean most } \\
\text { significant for incontinence. } \\
\text { Dmean increase by } 33 \mathrm{~Gy} \\
\text { increased incontinence by } \\
12 \%\end{array}$ & $\begin{array}{l}1,2,4,6,7 \\
(\mathrm{n} / \mathrm{a} 3)\end{array}$ & 1,2 \\
\hline $\begin{array}{l}\text { Mavroidis } \\
{[27]}\end{array}$ & $\begin{array}{l}\text { Anal } \\
\text { sphincter } \\
\text { region }\end{array}$ & $\begin{array}{l}\text { Musculaure layer around } \\
\text { the rectal aperture, } 3 \mathrm{~cm} \\
\text { caudal from anal verge }\end{array}$ & $\begin{array}{l}\text { Own } \\
\text { questionnaire }\end{array}$ & $\begin{array}{l}\text { faecal leakage } \\
\text { 19/65 (29\%); } \\
\text { blood/mucus } \\
22 / 65(34 \%)\end{array}$ & $\begin{array}{l}\text { Relative seriality NTCP model } \\
\text { of anal sphincter for } \\
\text { incontinence, blood/mucus. } \\
\text { Parameters for incontinence: } \\
\text { D50=70.2Gy, } \gamma=1.22, s= \\
0.35 . \text { Parameters for blood/ } \\
\text { mucus: } D 50=74.0 \mathrm{G}, y= \\
0.75, s \approx 0\end{array}$ & $\begin{array}{l}1,3,5,6,7 \\
(\mathrm{n} / \mathrm{a} 2)\end{array}$ & 1,3 \\
\hline Peeters [28] & $\begin{array}{l}\text { Anal } \\
\text { canal wall }\end{array}$ & $\begin{array}{l}\text { Wall of caudal } 3 \mathrm{~cm} \text { of } \\
\text { anorectum (method } \\
\text { described) }\end{array}$ & $\begin{array}{l}\text { Incontinence } \\
\text { requiring pad } \\
\text { use }>2 \text { x/wk.; }\end{array}$ & 32/368 (7\%) & $\begin{array}{l}\text { NTCP LKB model of } \\
\text { incontinence with anal wall } \\
\text { dose. Parameters found were } \\
n=7.48 ; \text { TD } 50=105 ; \mathrm{m}= \\
0.46\end{array}$ & $\begin{array}{l}\text { 1,3,4,5,6,7 } \\
\text { (n/a: } 2)\end{array}$ & 1,3 \\
\hline $\begin{array}{l}\text { Smeenk } \\
{[26]}\end{array}$ & $\begin{array}{l}\text { Anal } \\
\text { sphincter } \\
\text { muscles }\end{array}$ & $\begin{array}{l}\text { Individual muscles } \\
\text { defined (Internal anal } \\
\text { sphincter (IAS), external } \\
\text { anal sphincter (EAS), } \\
\text { puborectalis \& levator } \\
\text { ani) }\end{array}$ & $\begin{array}{l}\text { Frequency, } \\
\text { Urgency, } \\
\text { Incontinence }\end{array}$ & 21/48 (44\%) & $\begin{array}{l}\text { For complication }<5 \% \\
\text { Dmean<30Gy to IAS; }<10 G y \\
\text { to EAS, }<50 G y \text { to } \\
\text { puborectalis, }<40 G y \text { to } \\
\text { levator ani }\end{array}$ & $\begin{array}{l}1,4,5 \\
(\mathrm{n} / \mathrm{a} 2)\end{array}$ & $1,2,3$ \\
\hline $\begin{array}{l}\text { Smeenk } \\
\text { [22] }\end{array}$ & Anal wall & $\begin{array}{l}\text { Continuation of rectal } \\
\text { wall from anal verge to } \\
\text { slice below lowest slice } \\
\text { with a rectal balloon }\end{array}$ & $\begin{array}{l}\text { Frequency, } \\
\text { urgency, } \\
\text { incontinence }\end{array}$ & $\begin{array}{l}39 \% \\
\text { frequency, } \\
31 \% \text { urgency, } \\
31 \% \\
\text { incontinence }\end{array}$ & $\begin{array}{l}\text { For urgency: } \\
\text { Anal wall Dmean }<38 \text { Gy risk } \\
<15 \% \text {, >38Gy risk is } 62 \%\end{array}$ & $\begin{array}{l}1,4,7 \\
(\mathrm{n} / \mathrm{a} 2,3,5,6)\end{array}$ & 1,3 \\
\hline Lind [12] & $\begin{array}{l}\text { Anal } \\
\text { sphincter } \\
\text { region }\end{array}$ & $\begin{array}{l}\text { Inner muscle layer of the } \\
\text { sphincter up to anal } \\
\text { verge }\end{array}$ & $\begin{array}{l}\text { Defecation into } \\
\text { clothing } \\
\text { without } \\
\text { warning }>1 \text { in } \\
\text { last } 6 \text { months }\end{array}$ & $\begin{array}{l}63 / 519 \\
(12.1 \%)\end{array}$ & $\begin{array}{l}\text { Mean dose }>50 G y \text { to small } \\
\text { bowel or sigmoid or anal } \\
\text { sphincter region associated } \\
\text { with this symptom } \\
\text { (findings for individual } \\
\text { organs not clarified) }\end{array}$ & 1,7 (n/a 2,3) & $1,2,3$ \\
\hline Yeoh [24] & Anal wall & $\begin{array}{l}\text { From anorectal junction } \\
\text { (not clearly defined) }\end{array}$ & $\begin{array}{l}\text { LENT-SOMA } \\
\text { total score }\end{array}$ & $72 \%$ & $\begin{array}{l}\text { Anal wall } \mathrm{V} 40>65 \% \\
\text { associated with chronic } \\
\text { toxicity. }\end{array}$ & 1,5 (n/a 2,3) & 2,3 \\
\hline Thor [29] & $\begin{array}{l}\text { Anal } \\
\text { sphincter }\end{array}$ & $\begin{array}{l}\text { Anal canal, inner and } \\
\text { outer sphincter (not } \\
\text { clearly defined) }\end{array}$ & $\begin{array}{l}\text { Questionnaire } \\
\text { of } 19 \text { questions } \\
\text { in } 4 \text { domains: } \\
\text { pain urgency, } \\
\text { mucus \& } \\
\text { incontinence. }\end{array}$ & $\begin{array}{l}\text { Specific to } \\
\text { each of } 19 \\
\text { question }\end{array}$ & $\begin{array}{l}5 \text { LKB models proposed for } \\
\text { anal sphincter doses. } \\
\text { Low anal sphincter dose } \\
\text { associated with faecal } \\
\text { leakage and pain. High anal } \\
\text { sphincter dose associated } \\
\text { with leakage. }\end{array}$ & $1,3,6$ (n/a 2) & $1,2,3$ \\
\hline Ebert [35] & $\begin{array}{l}\text { Anal } \\
\text { Canal }\end{array}$ & $\begin{array}{l}\text { Caudal } 3 \mathrm{~cm} \text { of } \\
\text { anorectum }\end{array}$ & $\begin{array}{l}\text { LENT-SOMA - } 8 \\
\text { symptoms }\end{array}$ & $\begin{array}{l}\text { Specific to } \\
\text { each } \\
\text { symptom }\end{array}$ & $\begin{array}{l}\text { Bleeding associated with } \\
>40 \mathrm{~Gy} \text {, proctitis with 36- } \\
63 \mathrm{~Gy} \text {, frequency with 8-85Gy, } \\
\text { urgency and tenesmus with }\end{array}$ & $1,5,7$ (n/a 2) & $1,2,3$ \\
\hline
\end{tabular}


Table 4 Large Bowel studies - details and quality assessment (Continued)

\begin{tabular}{|c|c|c|c|c|c|c|c|}
\hline \multirow[b]{2}{*}{ Author } & \multirow[b]{2}{*}{$\begin{array}{l}\text { OAR } \\
\text { studied }\end{array}$} & \multirow[b]{2}{*}{ OAR defined } & \multirow[b]{2}{*}{$\begin{array}{l}\text { Toxicity } \\
\text { definition }\end{array}$} & \multirow[b]{2}{*}{$\begin{array}{l}\text { Pts with } \\
\text { toxicity }\end{array}$} & \multirow[b]{2}{*}{ Significant findings } & \multicolumn{2}{|c|}{ Quality Assessment } \\
\hline & & & & & & $\begin{array}{l}\text { Statistical } \\
\text { considerations } \\
\text { met }(1-7)\end{array}$ & $\begin{array}{l}\text { Endpoint } \\
\text { considerations } \\
\text { met }(1-3)\end{array}$ \\
\hline
\end{tabular}

Abbreviations: Pts Patients, OAR Organs at risk, RT Radiotherapy, Gr Grade, CTCAE Common terminology criteria for adverse events, RTOG Radiation therapy oncology group, LENT-SOMA Late Effects of Normal Tissue - Subjective Objective Management Analytical, RILIT Radiation induced late intestinal toxicity, EORTC European Organisation for Research and Treatment of Cancer, Vx Volume receiving x Gy, AUC Area under curve, Dmean Mean dose, Dmax Maximal dose, DVH Dose volume histogram, DSH Dose surface histogram, NTCP Normal Tissue Complication Probability, LKB Lyman Kutcher Burman

[27] modelled dose to the anal sphincter region for 'faecal leakage' and 'blood or phlegm' in stools using the relative seriality NTCP model. They recommended a reduction in the biologically effective uniform dose (EUD) to anal sphincter $<40-45$ Gy may significantly reduce toxicity.

\section{Quality assessment \\ Statistical criteria}

Most studies provided information on basic statistical data (29/30) and gave clear definitions of OARs (24/30). Constraints were derived in 16 papers, with associated complication rates stated in 12 papers. Goodness-of-fit was reported in 6 studies, with discriminator statistics reported in 10 papers. For the 4 papers with NTCP models all provided parameter estimates with standard error.

\section{Endpoint criteria}

Overall toxicity grades rather than individual symptoms were assessed in 13 of 30 studies, with patient-reported outcomes used in 14 studies (13 of which were studies of the anal canal). 21 of the studies looked at co-morbidity to assess its contribution to late toxicity and this was taken into account in multivariate analyses if thought to be associated.

\section{Discussion}

We have systematically reviewed the currently published literature on dose-volume constraints for late bowel toxicity after pelvic radiotherapy, excluding the rectum. We identified 30 studies including 5136 patients. A key finding was consistent dose-volume constraints defined for the anal canal from five studies. For whole bowel loops, small bowel, duodenum, large bowel and sigmoid dose-volume constraints were derived in individual studies, however there was limited validation of these findings in other studies examining the same component of bowel.

Of all the components of bowel studied, most data were available in the 16 studies examining the anal canal or anal sphincter region, and these studies were most conclusive. Statistical and endpoint measures recommended by QUANTEC were met much more frequently in these studies, data of which originated mainly from prostate clinical trials. Fifteen of these sixteen studies used individual symptoms reported by patients rather than an overall toxicity score.

These studies clearly indicate a relationship between dose-volume parameters to the anal canal and faecal incontinence. Dmean was the most significant parameter in five different studies, with a range of doses between $40-47 \mathrm{~Gy}$ found. From the available data we recommend a constraint Dmean of <40Gy (in 2Gy fractions) to the anal canal to be included in clinical protocols in order to limit late bowel toxicity, in particular faecal incontinence.

For other components of bowel, the evidence was far less conclusive, as the findings of single studies were not corroborated with others. Possible reasons could be

Table 5 Anal canal Dmean results

\begin{tabular}{|c|c|c|c|c|c|c|}
\hline Study & $\begin{array}{l}\text { No of } \\
\text { pts }\end{array}$ & OAR & Endpoint & $\begin{array}{l}\text { Dmean (in EQD2) } \\
\text { constraint }\end{array}$ & $\begin{array}{l}\text { Risk of endpoint below this } \\
\text { constraint }\end{array}$ & $\begin{array}{l}\text { Risk of endpoint above this } \\
\text { constraint }\end{array}$ \\
\hline $\begin{array}{l}\text { Al-albany } \\
{[18]}\end{array}$ & 65 & $\begin{array}{l}\text { Anal sphincter } \\
\text { region }\end{array}$ & Incontinence $>2 X /$ week & 43.2 & $8 \%$ & $52 \%$ \\
\hline $\begin{array}{l}\text { Alsadius } \\
\text { [19] }\end{array}$ & 403 & Anal canal & Incontinence $>1 \mathrm{x} /$ month & 40 & $5.2 \%$ & $21 \%$ \\
\hline $\begin{array}{l}\text { Buettner } \\
{[20]}\end{array}$ & 388 & $\begin{array}{l}\text { Anal sphincter } \\
\text { region }\end{array}$ & $\begin{array}{l}\text { Incontinence: moderate/ } \\
\text { severe (gr2) }\end{array}$ & $\begin{array}{l}47 \text {, though }<30 G y \\
\text { ideal }\end{array}$ & $\begin{array}{l}\text { 5\% (approx; read from } \\
\text { graph) }\end{array}$ & \\
\hline $\begin{array}{l}\text { Smeenk } \\
{[22]}\end{array}$ & 36 & Anal canal wall & Urgency present & 41.8 & $15 \%$ & $62 \%$ \\
\hline $\begin{array}{l}\text { Peeters } \\
{[21]}\end{array}$ & 641 & Anal canal wall & $\begin{array}{l}\text { Incontinence requiring pad } \\
>2 x / \text { week }\end{array}$ & $\begin{array}{l}\text { No constraint } \\
\text { specified }\end{array}$ & $16 \%$ at $19 \mathrm{~Gy}$ & $31 \%$ at $52 \mathrm{~Gy}$ \\
\hline
\end{tabular}


differences in the endpoint studied (i.e toxicity, grade and clinician versus patient-reporting) and differences in the definition of the OARs. Furthermore different studies derive constraints with different aims, with some using constraints to lower the risk of toxicity to a certain level eg. to $5 \%$ or to $20 \%$, and others attempt to derive constraints with the aim of no toxicity at all.

For example when considering constraints for bowel loops, both Guerrero-Urbano et al. [8] and McDonald et al. [9] derived constraints for V40, V45 and V60 associated with late bowel toxicity. However, the constraints in these studies differed, with one suggesting $\mathrm{V} 40<124 \mathrm{cc}, \mathrm{V} 45<$ $71 \mathrm{cc}$ and $\mathrm{V} 60<0.5 \mathrm{cc}$, and the other recommending V40 $<151 \mathrm{cc}, \mathrm{V} 45<139 \mathrm{cc}$ and $\mathrm{V} 60<98 \mathrm{cc}$, despite the same definition of bowel loops, and use of RTOG scoring. Reasons for these differences could be due to differences in endpoint definition, with one study looking specifically at $\geq$ grade 2 RTOG diarrhoea, with the other looking at overall RTOG toxicity $\geq$ grade 1 . McDonald et al. determined constraints aiming to reduce the risk of $\geq$ grade 1 toxicity specifically to less than $25 \%$, whereas Guerrero-Urbano et al. found constraints with the aim of reducing $\geq$ grade 2 toxicity, but the level to which this aims to reduce the risk of toxicity is unclear.

A similar lack of consistency was seen for studies focussed on duodenum [13,30], large bowel [10] and sigmoid colon [6, 17] making it difficult to further recommend constraints for these OARs. Future validation of the published constraints using independent data sets from patients using the same toxicity endpoints, same OAR definitions and the same aim in terms of toxicity reduction, would be a useful next step to improve knowledge on this subject.

Many studies found no correlation with OAR dose parameters and late bowel toxicity at all. This lack of findings could be due to a variety of methodological reasons - many of the studies were underpowered with only a very small incidence of the defined toxicity, making it difficult to determine the likely predictors of these toxicities in only a handful of patients. Many studies have not collected baseline data and presume the presence of bowel symptoms post-radiotherapy is treatment related, when in fact these symptoms may have been pre-existing, or due to a separate bowel pathology. Further a known issue within the pelvis, is that of organ motion of bowel and its subsection, and the use of a single CT scan to define a highly mobile structure may not be an appropriate approach.

Aside from methodology the reason for lack of positive findings may be in fact that particular OARs may genuinely not have any influence on late toxicity, and rather than dose-volume predictors, other considerations such as inherent radiosensitivity of individual patients, may be the main predictors of toxicity.
For acute bowel toxicity QUANTEC have suggested two constraints: V45 < 195 cc for peritoneal cavity, and $\mathrm{V} 15<120 \mathrm{cc}$ for small bowel loops. The QUANTEC authors suggest these constraints may be applicable for late bowel toxicity. Some consistency is seen to QUANTEC recommendations within this review with Chopra et al. [11] finding V15 small bowel loops to be important on multivariate analysis, although their recommended constraint was much higher at V15 $<275 \mathrm{cc}$.

For peritoneal cavity, the findings of the studies reviewed do not corroborate with QUANTEC. Three studies found no correlation of peritoneal cavity doses with late toxicity, and the two positive studies found that in fact lower doses to peritoneal cavity of V20 and V10$30[6,31]$ were predictive of late toxicity. It would be important to validate the significance of these low doses in terms of late toxicity given the increased use of volumetric arc therapy (VMAT) techniques in recent years, where lower dose bath to a larger area of normal tissue is seen, the significance of which is currently not understood.

Strengths of this systematic review are the broad inclusivity of the search, with the studies included having patients with different tumour types, radiotherapy techniques, fractionations, and concurrent treatments. A similar approach was used in key papers such as the Emami et al. data [32], as well as the QUANTEC papers $[3,4]$, where bowel constraints were sought from studies with gynaecological, rectal, prostate and pancreatic cancers. A potential limitation of this is that some of these treatment factors may influence late toxcity (e.g. use of concurrent systemic agents, or hypofractionation). Although it is expected that individual authors may account for these factors statistically this may not have always been done and may explain partly the inconsistent results found.

Despite attempting to be as inclusive as possible we may have missed those studies not in English, and from grey literature currently unpublished. Studies involving SBRT were excluded given the questionable validity of the linear quadratic model with extreme hypofractionation thus making radiobiological comparisons difficult [33].

Quality assessment based on the QUANTEC-defined criteria added much value to this review, highlighting that many researchers do not report or consider the endpoint or statistical criteria, and further that those that do adhere to these criteria appear to have more conclusive findings.

\section{Conclusions}

We recommend the use of Dmean to the anal canal of $<40$ Gy in pelvic radiotherapy protocols as a constraint to reduce the development of late bowel toxicity, in particular faecal incontinence. 
Other important organs at risk to consider are whole bowel loops, small bowel, duodenum, large bowel and sigmoid colon and constraints for these OARS are noted in this review. However, clear recommendations for these organs cannot be made, due to lack of correlation between studies. Validation of the constraints found within this systematic review for these OARS with independent data sets would be an important next step. If validated these constraints could be used clinically in prospective patients, and also as a relevant benchmark to assess the likely impact of advanced radiotherapy techniques on late toxicity. Future studies should consider the quality criteria recommended by QUANTEC.

\begin{abstract}
Abbreviations
Cc: Cubic centimetres; CTCAE: Common Terminology Criteria Adverse Events; Dmean: Mean Dose; DSH: Dose Surface Histogram; DVH: Dose Volume Histogram; EUD: Equivalent Uniform Dose; Gy: Gray; LKB: Lyman-Kutcher Burman; OAR: Organ at Risk; QUANTEC: Quantitative Analysis of Normal Tissue Effects in the Clinic; RTOG: Radiation Therapy Oncology Group: VMAT: Volumetric Modulated Arc Therapy; Vx Gy: Volume receiving x Gray
\end{abstract}

\section{Acknowledgements}

Not applicable.

\section{Funding}

This work was supported by Velindre Cancer Centre Charitable Funds, and Cancer Research Wales.

\section{Availability of data and materials}

Data sharing not applicable to this article as no datasets were generated or analysed during the current study.

\section{Authors' contributions}

BC performed all systematic searching. RJ and EH screened all abstracts, and scrutinized relevant full texts and hand-searched for additional references. All authors read and approved the final draft of this manuscript.

\section{Ethics approval and consent to participate}

Not applicable.

\section{Consent for publication}

Not applicable.

\section{Competing interests}

The authors declare they have no competing interests.

\section{Publisher's Note}

Springer Nature remains neutral with regard to jurisdictional claims in published maps and institutional affiliations.

\section{Author details}

${ }^{1}$ Department of Clinical Oncology, Velindre Cancer Centre, Velindre Road, Whitchurch, Cardiff CF14 2TL, UK. '2Department of Clinical Oncology, Addenbrookes' Hospital, Box 193, Cambridge CB2 0QQ, UK. ${ }^{3}$ Cancer Research Wales Library, Velindre Cancer Centre, Velindre Road, Whitchurch, Cardiff CF14 2TL, UK. ${ }^{4}$ School of Medicine, Institute of Cancer and Genetics, Cardiff University, Velindre Cancer Centre, Velindre Road, Whitchurch, Cardiff CF14 2TL, UK.

Received: 7 May 2018 Accepted: 27 March 2019

Published online: 03 April 2019

\section{References}

1. Benton B, Norton C, Lindsay JO, Dolan S, Andreyev HJ. Can nurses manage gastrointestinal symptoms arising from pelvic radiation disease? Clin Oncol (R Coll Radiol). 2011;23(8):538-51.
2. Andreyev HJ. Gastrointestinal problems after pelvic radiotherapy: the past, the present and the future. Clin Oncol (R Coll Radiol). 2007;19(10):790-9.

3. Michalski JM, Gay H, Jackson A, Tucker SL, Deasy JO. Radiation dose-volume effects in radiation-induced rectal injury. Int J Radiat Oncol Biol Phys. 2010; 76(3 Suppl):S123-9.

4. Kavanagh BD, Pan CC, Dawson LA, Das SK, Li XA, Ten Haken RK, et al. Radiation dose-volume effects in the stomach and small bowel. Int J Radiat Oncol Biol Phys. 2010;76(3 Suppl):S101-7.

5. Jackson A, Marks LB, Bentzen SM, Eisbruch A, Yorke ED, Ten Haken RK, et al. The lessons of QUANTEC: recommendations for reporting and gathering data on dose-volume dependencies of treatment outcome. Int J Radiat Oncol Biol Phys. 2010;76(3 Suppl):S155-60.

6. Mouttet-Audouard R, Lacornerie T, Tresch E, Kramar A, Le Tinier F, Reynaert $\mathrm{N}$, et al. What is the normal tissues morbidity following helical intensity modulated radiation treatment for cervical cancer? Radiother Oncol. 2015; 115(3):386-91.

7. Deville C, Vapiwala N, Hwang WT, Lin H, Ad VB, Tochner Z, et al. Comparative toxicity and dosimetric profile of whole-pelvis versus prostate bed-only intensity-modulated radiation therapy after prostatectomy. Int J Radiat Oncol Biol Phys. 2012;82(4):1389-96.

8. Guerrero Urbano T, Khoo V, Staffurth J, Norman A, Buffa F, Jackson A, et al. Intensity-modulated radiotherapy allows escalation of the radiation dose to the pelvic lymph nodes in patients with locally advanced prostate cancer: preliminary results of a phase I dose escalation study. Clin Oncol (R Coll Radiol). 2010;22(3):236-44.

9. McDonald F, Waters R, Gulliford S, Hall E, James N, Huddart RA. Defining bowel dose volume constraints for bladder radiotherapy treatment planning. Clin Oncol (R Coll Radiol). 2015;27(1):22-9.

10. Isohashi F, Yoshioka Y, Mabuchi S, Konishi K, Koizumi M, Takahashi Y, et al. Dose-volume histogram predictors of chronic gastrointestinal complications after radical hysterectomy and postoperative concurrent nedaplatin-based chemoradiation therapy for early-stage cervical cancer. Int J Radiat Oncol Biol Phys. 2013;85(3):728-34.

11. Chopra S, Dora T, Chinnachamy AN, Thomas B, Kannan S, Engineer R, et al. Predictors of grade 3 or higher late bowel toxicity in patients undergoing pelvic radiation for cervical cancer: results from a prospective study. Int J Radiat Oncol Biol Phys. 2014;88(3):630-5.

12. Lind $H$, Alevronta $E$, Steineck $G$, Waldenstrom AC, Nyberg T, Olsson C, et al. Defecation into clothing without forewarning and mean radiation dose to bowel and anal-sphincter among gynecological cancer survivors. Acta Oncol. 2016;55(11):1285-93.

13. Kelly P, Das P, Pinnix CC, Beddar S, Briere T, Pham M, et al. Duodenal toxicity after fractionated chemoradiation for unresectable pancreatic cancer. Int J Radiat Oncol Biol Phys. 2013;85(3):143-9.

14. Fokdal $\mathrm{L}$, Honore $\mathrm{H}$, Hoyer $\mathrm{M}$, von der Maase $\mathrm{H}$. Dose-volume histograms associated to long-term colorectal functions in patients receiving pelvic radiotherapy. Radiother Oncol. 2005;74(2):203-10.

15. Huang J, Roberson JM, Ye H, Yan D. Dose-volume analysis of predictors for gastrointestinal toxicity after radiotherapy and concurrent fulldose gemcitabine for locally advanced pancreatic adenocarcinoma. Int J Radiat Oncol Biol Phys. 2011;83(4):1120-5.

16. Poorvu PD, Sadow CA, Townamchai K, Damato AL, Viswanathan AN. Duodenal and other gastrointestinal toxicity in cervical and endometrial cancer treated with extended-field intensity modulated radiation therapy to paraaortic lymph nodes. Int J Radiat Oncol Biol Phys. 2013;85(5):1262-8.

17. Fonteyne V, De Neve W, Villeirs G, De Wagter C, De Meerleer G. Late radiotherapy-induced lower intestinal toxicity (RILIT) of intensity-modulated radiotherapy for prostate cancer: the need for adapting toxicity scales and the appearance of the sigmoid colon as co-responsible organ for lower intestinal toxicity. Radiother Oncol. 2007;84(2):156-63.

18. al-Abany $M$, Helgason AR, Cronqvist AK, Lind $B$, Mavroidis $P$, Wersall $P$, et al. Toward a definition of a threshold for harmless doses to the anal-sphincter region and the rectum. Int J Radiat Oncol Biol Phys. 2005;61(4):1035-44.

19. Alsadius D, Hedelin M, Lundstedt D, Pettersson N, Wilderang U, Steineck G. Mean absorbed dose to the anal-sphincter region and fecal leakage among irradiated prostate cancer survivors. Int J Radiat Oncol Biol Phys. 2012;84(2):e181-5.

20. Buettner F, Gulliford SL, Webb S, Sydes MR, Dearnaley DP, Partridge M. The dose-response of the anal sphincter region--an analysis of data from the MRC RT01 trial. Radiother Oncol. 2012;103(3):347-52.

21. Peeters ST, Lebesque JV, Heemsbergen WD, van Putten WL, Slot A, Dielwart $M F$, et al. Localized volume effects for late rectal and anal toxicity after 
radiotherapy for prostate cancer. Int J Radiat Oncol Biol Phys. 2006;64(4): 1151-61.

22. Smeenk RJ, Hopman WP, Hoffmann AL, van Lin EN, Kaanders JH. Differences in radiation dosimetry and anorectal function testing imply that anorectal symptoms may arise from different anatomic substrates. Int I Radiat Oncol Biol Phys. 2012;82(1):145-52.

23. Vordermark D, Schwab M, Ness-Dourdoumas R, Sailer M, Flentje M, Koelbl O. Association of anorectal dose-volume histograms and impaired fecal continence after 3D conformal radiotherapy for carcinoma of the prostate. Radiother Oncol. 2003;69(2):209-14.

24. Yeoh EK, Krol R, Dhillon VS, Botten R, Di Matteo A. Butters J, et al... Predictors of radiation-induced gastrointestinal morbidity: a prospective, longitudinal study following radiotherapy for carcinoma of the prostate. Acta Oncol. 2016;55(5):604-10.

25. Koper PC, Jansen P, van Putten W, van Os M, Wijnmaalen AJ, Lebesque JV, et al. Gastro-intestinal and genito-urinary morbidity after 3D conformal radiotherapy of prostate cancer: observations of a randomized trial. Radiother Oncol. 2004;73(1):1-9.

26. Smeenk RJ, Hoffmann AL, Hopman WP, van Lin EN, Kaanders JH. Doseeffect relationships for individual pelvic floor muscles and anorectal complaints after prostate radiotherapy. Int J Radiat Oncol Biol Phys. 2012; 83(2):636-44.

27. Mavroidis P, al-Abany M, Helgason AR, Agren Cronqvist AK, Wersall P, Lind $H$, et al. Dose-response relations for anal sphincter regarding fecal leakage and blood or phlegm in stools after radiotherapy for prostate cancer. Radiobiological study of 65 consecutive patients. Strahlenther Onkol. 2005; 181(5):293-306.

28. Peeters ST, Hoogeman MS, Heemsbergen WD, Hart AA, Koper PC, Lebesque JV. Rectal bleeding, fecal incontinence, and high stool frequency after conformal radiotherapy for prostate cancer: normal tissue complication probability modeling. Int J Radiat Oncol Biol Phys. 2006;66(1):11-9.

29. Thor M, Olsson CE, Oh JH, Petersen SE, Alsadius D, Bentzen L, et al. Relationships between dose to the gastro-intestinal tract and patientreported symptom domains after radiotherapy for localized prostate cancer. Acta Oncol. 2015;54(9):1326-34

30. Verma J, Sulman EP, Jhingran A, Tucker SL, Rauch GM, Eifel PJ, et al. Dosimetric predictors of duodenal toxicity after intensity modulated radiation therapy for treatment of the Para-aortic nodes in gynecologic cancer. Int J Radiat Oncol Biol Phys. 2014;88(2):357-62.

31. Deville C, Both S, Hwang WT, Tochner Z, Vapiwala N. Clinical toxicities and dosimetric parameters after whole-pelvis versus prostate-only intensitymodulated radiation therapy for prostate cancer. Int J Radiat Oncol Biol Phys. 2010;78(3):763-72.

32. Emami B, Lyman J, Brown A, Coia L, Goitein M, Munzenrider JE, et al. Tolerance of normal tissue to therapeutic irradiation. Int J Radiat Oncol Biol Phys. 1991;21(1):109-22.

33. Kirkpatrick JP, Meyer JJ, Marks LB. The linear-quadratic model is inappropriate to model high dose per fraction effects in radiosurgery. Semin Radiat Oncol. 2008;18(4):240-3.

34. Adkison JB, McHaffie DR, Bentzen SM, Patel RR, Khuntia D, Petereit DG, et al. Phase I trial of pelvic nodal dose escalation with hypofractionated IMRT for high-risk prostate cancer. Int J Radiat Oncol Biol Phys. 2012;82(1):184-90.

35. Ebert MA, Foo K, Haworth A, Gulliford SL, Kennedy A, Joseph DJ, et al. Gastrointestinal dose-histogram effects in the context of dose-volumeconstrained prostate radiation therapy: analysis of data from the RADAR prostate radiation therapy trial. Int J Radiat Oncol Biol Phys. 2015;91(3):595-603.

36. Green GLA, Zhang J, Ramsinghani N, Asawi S. Dose-volume relationship of acute and late small bowel toxicity from radiation therapy for prostate cancer: a veterans affairs study. J Radiat Oncol. 2015;4:411-5.

37. Taussky D, Schneider U, Rousson V, Pescia R. Patient-reported toxicity correlated to dose-volume histograms of the rectum in radiotherapy of the prostate. Am J Clin Oncol. 2003;26(5):144-9.

Ready to submit your research? Choose BMC and benefit from:

- fast, convenient online submission

- thorough peer review by experienced researchers in your field

- rapid publication on acceptance

- support for research data, including large and complex data types

- gold Open Access which fosters wider collaboration and increased citations

- maximum visibility for your research: over $100 \mathrm{M}$ website views per year

At BMC, research is always in progress.

Learn more biomedcentral.com/submissions 